\title{
Le portfolio: une médiation contrôlante et structurante des savoirs professionnels
}

\section{Alexandre A.J. Buysse ${ }^{1}$ et Sabine Vanhulle}

Selon la perspective vygotskienne qui est la nôtre, le portfolio est un outil qui permet une médiation non seulement du contrôle des apprentissages, mais également une médiation d'une procédure liée aux savoirs. Nous voyons ainsi le portfolio comme une médiation contrôlante et structurante. Nous examinons comment il permettrait de rendre visible dans un premier temps et d'intérioriser dans un deuxième temps le processus de création des savoirs professionnels issus des savoirs de référence et des savoirs expérientiels du sujet. Nous illustrons nos propos par une description de nos interventions-portfolios dans nos contextes d'instituts de formation à l'enseignement.

\section{Introduction}

Le portfolio est une collection systématique et organisée de travaux faisant l'objet d'une réflexion. Le portfolio permet ainsi de comprendre les forces des sujets, leurs processus d'apprentissage, leurs progrès et leurs besoins de formation. Il atteste également de leur développement professionnel et des compétences atteintes (Antonek, 1997; Costantino, De Lorenzo \& Kobrinski, 2002; Foster, Walker \& Song, 2007; Paris \& Ayres, 1994; Vanhulle \& Schillings, 2004). Souvent, des buts de présentation, de certification et d'apprentissage sont visés en même temps. La sélection impliquée dans la collection des traces exige déjà une certaine réflexivité afin de pouvoir justifier les choix effectués. La plupart du temps, il ne s'agit que du premier pas vers un travail réflexif plus intense.

Dans notre perspective, l'instrument privilégié des portfolios d'apprentissage et de développement est en effet l'écriture "réflexive». Suscitée tout au long du temps d'apprentissage professionnel, elle s'appuie sur les traces commentées et parfois réorganisées au fil du temps (Vanhulle, 2005, 2009b). La collection de traces finit dans ce cas par être une sorte d'annexe documentaire au portfolio luimême (Doolittle, 1994). Les portfolios de développement professionnel accordent ainsi une place prépondérante à une réflexivité écrite. Dans leur cas, tout en intégrant le retour sur soi et ses actions, tout en respectant les dimensions 
affectives qui entrent dans l'apprentissage, le cœur de cette réflexivité et de la mise en discours écrit qu'elle soutient concerne la production par les étudiants de savoirs professionnels. Cette construction réflexive et discursive se situe entre:

- une construction personnalisée par le sujet de significations qui lui permettent de donner sens à ses actions actuelles et futures en lien avec ces savoirs professionnels,

- et une adéquation des savoirs professionnels construits par rapport aux attentes de la formation de base et de la profession.

Les portfolios peuvent ainsi être considérés comme des instruments réflexifs de nature mixte, combinant à la fois la collection systématique - propre au portfolio artistique qui est à son origine - et l'écriture réflexive.

Cette réflexivité écrite peut être guidée ou non par des questionnements, préalable ou non à des échanges avec des formateurs, reliée ou non avec des pairs (Deum \& Vanhulle, 2008; Vanhulle \& Schillings, 2004). Il nous semble donc essentiel d'interroger la finalité de l'écriture réflexive et de ses fonctions en termes de construction - et d'intériorisation - des savoirs. En effet, de nombreux dispositifs portfolio s'appuient sur la volonté de satisfaire à une exigence de certification ou de reconnaissance obéissant à une logique critériée dans l'esprit des compétences. Mais si le portfolio veut susciter également des démarches d'autoévaluation et d'autodéveloppement chez l'apprenant, alors nous devons considérer que l'écriture réflexive amène autre chose qu'une simple démonstration de compétences acquises (Vanhulle, 2009c). Même si démonstration il y a, pour être authentique, celle-ci ne saurait se fonder que sur le développement de savoirs propres au sujet.

Ceci nous amène, avant de revenir sur les fonctions de l'écriture comme outil de développement, à saisir la teneur particulière des savoirs professionnels, et cela en prenant en compte l'impact de la formation à l'enseignement en alternance sur ces savoirs.

\section{L'émergence de savoirs professionnels entre apprentissage et développement}

Les dispositifs de formation en alternance proposent: lors des cours en institution, des savoirs de référence, qui sont externes au sujet et qui disposent de leur propre épistémie; des situations de stages en milieu de travail, où l'étudiant peut créer des savoirs issus de ses expériences propres tout en se confrontant aux pratiques en vigueur dans ces milieux. Ces dispositifs exigent in fine que l'étudiant crée ses propres savoirs professionnels sur la base de ces savoirs d'origines totalement différentes (Vanhulle, 2008, 2009c, 2009d).

Nous posons ainsi que les savoirs professionnels construits par le sujet constituent des énoncés, formalisés dans un entrecroisement complexe d'offres de significations circulant dans l'alternance: entre des savoirs de référence pour la 
profession (savoirs académiques-scientifiques, savoirs prescrits par l'institution éducative, savoirs transmis par des formateurs sur le terrain) et des savoirs construits à partir de l'expérience en propre (Vanhulle, sous presse). Les savoirs professionnels sont ainsi le résultat de l'intériorisation car ils sont liés intrinsèquement au sens propre que donne l'apprenant.

Les enseignants, tant novices qu'experts, élaborent en effet des changements dans leurs théories en passant par: une réflexion sur leurs propres conceptions, une problématisation de leur pratique et d'éventuels changements à leurs théories et pratiques (Fenstermacher, 1994). Le savoir est ici conçu comme étant utilisé consciemment en ayant recours à des techniques d'investigation pour pouvoir faire face à de nouvelles situations (Wood \& Bennett, 2000). Les savoirs pratiques peuvent ainsi être vus comme des manières personnelles de chaque professionnel de reconstruire le passé et d'élaborer des intentions futures afin de faire face aux exigences de la situation actuelle.

De ce fait, le savoir professionnel n'est pas immuable ni indépendant de la personne, c'est au contraire le résultat d'une action réfléchie et discriminante. Si le simple fait de faire une observation, d'agir ou de vivre une expérience est suffisant pour se former une opinion, ou une croyance, c'est néanmoins insuffisant pour construire un savoir. Dans ce but, le sujet doit faire preuve de réflexivité. Ceci permet, pour autant qu'un sens soit donné à l'expérience, d'induire un savoir de l'expérience qui pourra être transmis comme savoir pratique. Mais ce savoir pratique ne saurait à lui seul suffire pour créer un savoir admis en tant qu'énoncé valide dans le cadre des communautés discursives des sciences sociales ou naturelles (Fenstermacher \& Sanger, 1998), auxquelles nous devons ajouter les communautés professionnelles qui entretiennent des liens avec celles-ci. A cette fin, il convient de compléter ce savoir de l'expérience par des savoirs de référence.

Prendre le contrôle de ses apprentissages implique ainsi une réflexivité sur sa pratique dépassant la simple conscience de ce qui a été réalisé. Il s'agit de faire preuve d'une réflexivité sur les activités et leur cadre de référence théorique ainsi que d'une capacité à les articuler à d'autres savoirs d'une manière qui fait sens. On peut donc fournir aux étudiants des moyens de relier les différents cadres de référence à leur activité de travail. Ceci leur permet d'être plus conscients de leurs propres conceptions et des motifs, justifications et intentions qui entrent nécessairement dans tout savoir professionnel.

Dans cette optique, les savoirs professionnels créés sous l'impact de pratiques réflexives fines relient l'acquisition adéquate de savoirs de référence, l'analyse des situations rencontrées dans des contextes situés, la refiguration des expériences vécues et des croyances personnelles.

Soulevons à cet égard que la pratique réflexive elle-même, tout en étant une compétence transversale reliée à une forme particulière de la pensée et de l'agir humains, est nécessairement reliée aux contextes, situations, normes, valeurs et savoirs faire d'une profession définie (Schön, 1983, 1987), donc à une culture 
professionnelle donnée (Buysse, sous presse b). Derrière tout savoir culturellement transmis existe ainsi une structuration interne, propre à son élaboration sociale, une épistémie inscrite dans une culture disciplinaire dans laquelle le savoir s'inscrit (Buysse, 2009) - pour les enseignants en particulier, cela implique un travail d'appropriation de ce qui fonde leur métier: enseigner des savoirs et les faire apprendre (Hofstetter \& Schneuwly, 2009). Une telle épistémie se répercute dans l'approche même de la résolution de problèmes spécifiques à la profession donnée. Il existe une manière de faire, une épistémie professionnelle, transmise dans le milieu socioculturel de chaque profession et dans les établissements de formation qui y préparent (Buysse, sous presse, a, b). Profondément liée à la nature de l'activité, cette médiation est propre et discernable dans le discours des professionnels sur leur activité (Buysse \& Vanhulle, 2009). Il en ressort que les portfolios ne peuvent se concevoir en dehors de leur contexte socioculturel.

Nous posons donc l'existence d'une structure propre à l'élaboration des savoirs professionnels. Si celle-ci est personnelle et subjective, elle entretient en même temps des liens avec les caractéristiques de l'activité professionnelle en question. Nous pensons que cette structure peut être transmise par un portfolio conçu comme un dispositif d'ensemble qui implique des réélaborations discursives. Le dispositif portfolio propose alors un processus balisé à travers lequel le sujet pense sa profession. Une fois intériorisé, ce processus devient le moteur qui permet au sujet de créer ses propres savoirs professionnels, résultats à la fois d'une objectivation et d'une subjectivation (Buysse \& Vanhulle, 2009; Vanhulle, 2009a).

Plus subtilement, tout dispositif de formation représente ainsi à notre avis un jeu de médiations visant des processus d'intériorisation des savoirs par le sujet (Buysse, 2009).

\section{Médiations}

Dans une perspective vygotskienne, il ne saurait y avoir d'intériorisation de savoirs sans une médiation (Vygotsky, 1978). La médiation assure le passage du savoir de l'interpsychique à l'intrapsychique mais également la poursuite de l'intériorisation au niveau intrapsychique qui est liée à la création d'un sens propre à l'apprenant, intégré à son système psychologique et dès lors développemental (Vygotsky, 1997).

On peut distinguer sur cette base deux types de médiations permettant la poursuite de l'intériorisation d'un point de vue développemental (Buysse, 2009), en posant que:

1. Les médiations contrôlantes permettent au sujet de déterminer ce qui est sous son contrôle ou sous le contrôle de son environnement. Elles sont impliquées dans le transfert au sujet du contrôle de l'apprentissage et de l'évaluation. Ces médiations touchent à la réflexivité contrôlante ${ }^{2}$ : intégration d'objectifs, 
contrôle des régulations de ses manières de penser et de faire, adéquation aux exigences de l'évaluation. Ces médiations sont souvent liées directement à la communication et aux exigences du formateur. En intériorisant ces exigences, le sujet modifie son fonctionnement. A travers la poursuite de l'intériorisation des médiations contrôlantes, le sujet évolue vers des apprentissages plus ou moins autonomes, pouvant aller jusqu'à l'autoévaluation, une motivation intrinsèque et la détermination d'objectifs à atteindre 3 .

2. Les médiations structurantes sont, à notre avis, au cour de la perspective vygotskienne. Elles ont leur origine, entre autres, dans les concepts euxmêmes, dans le sens qui est donné aux savoirs. Grâce aux médiations structurantes, les significations culturellement établies sont transformées dans un travail subjectif de création de sens. Lors de l'intériorisation, ces médiations permettent le remplacement d'une structure externe propre aux savoirs par un agencement intrapsychique de ces savoirs. Lors de leur intériorisation, les concepts fournissent ainsi au sujet les outils cognitifs nécessaires à la compréhension des savoirs. Idéalement, les concepts scientifiques reflètent ce qui est interprété comme l'essence d'un certain aspect de la réalité, tout en ayant un caractère explicite, un système d'organisation systémique, logique et hiérarchique (Van der Veer, 1998). Lors de la poursuite de leur intériorisation, et en tension avec les concepts quotidiens du sujet, ils constituent un levier de développement de la pensée et de l'agir (Karpov, 2003; Karpov \& Haywood, 1998). La médiation structurante amènerait ainsi le sujet à prendre en compte différents savoirs dans l'examen de ses régulations et donc à faire preuve de réflexivité structurante ${ }^{4}$ dans le sens de la prise en compte de différents savoirs et de l'établissement des liens entre ceux-ci.

Ces médiations contrôlantes et structurantes sont fondées sur des outils et des signes qui contraignent et donnent au sujet des moyens de penser, d'agir et de réfléchir. A ce titre, les portfolios de développement professionnel sont des outils sémiotiques culturellement élaborés.

A notre avis, l'intériorisation de la médiation du portfolio influence le contrôle que prennent les apprenants sur leur processus de formation et sur le développement de leur identité professionnelle (Berrill \& Whalen, 2007). Le portfolio peut en effet susciter une réflexivité contrôlante qui conduit à la régulation, voire à l'autorégulation, de buts, de procédures, de manières d'atteindre ces buts. Ceci correspond à une médiation contrôlante. A notre avis, si le portfolio va jusqu’à infléchir le processus de régulation utilisé par le sujet luimême et ce qu'il prend en compte, non seulement dans une démarche réflexive, mais surtout dans un processus réflexif de transformation de sa pensée et de son agir, alors il peut également offrir une médiation structurante. Il peut aussi offrir des possibilités de développement d'une réflexivité structurante par un usage plus soutenu de concepts fondamentaux, en particulier dans la mise en discours de savoirs professionnels que l'énonciation écrite permet de réélaborer et de subjectiver par la création d'un sens. 
Le simple fait d'écrire ne contribue pas automatiquement à la création de sens. L'écriture peut améliorer les apprentissages si elle est au service de stratégies cognitives et métacognitives d'apprentissage autorégulé. Par exemple, le journal de bord de formation est une tâche exigeant certaines stratégies liées à l'écriture de ses propres réflexions sur les situations ou la matière vue. Ces stratégies incluent: l'organisation de la matière afin de questionner sa structure interne; l'élaboration de liens entre des savoirs d'origines différentes; l'usage volontaire de l'écriture comme un système de signes qui aide à penser, à structurer le savoir et à le transformer en significations propices à conceptualiser l'agir. Cet usage de l'écriture en quête de sens semble améliorer l'appropriation du savoir même audelà de ce qui est obtenu par des résumés ou des synthèses (Hübner, Nückels \& Renkl, 2009).

Nous pouvons ainsi considérer que les outils proposés par les dispositifs de type portfolio impliquent des phases de contrôle des opérations cognitives et discursives nécessaires à leur utilisation mais aussi des procédures particulières de régulation de leur utilisation (Buysse, 2008, octobre). Si nous admettons que leur utilisation peut être intériorisée, ces outils exercent dès lors une médiation à la fois contrôlante et structurante lors de l'intériorisation des savoirs qui leur sont liés. L'adéquation ou la complémentarité des outils aux savoirs ainsi mis en jeu est donc primordiale. Ceci nous amène à penser que, du point de vue de la médiation structurante, un portfolio ne saurait être «neutre». En effet, tout portfolio, dans la mesure où il comporte une visée formative, comprend plus qu'une simple incitation à une réflexion contrôlante et à une évaluation authentique ou une plus grande autonomie. Il invite le sujet à orienter ses apprentissages, à s'approprier les savoirs en fonction d'une certaine finalité éducative. Or, l'écriture semble souvent influencée par la volonté de faire des liens par exemple avec le référentiel de compétences, mais avant tout afin de réussir son évaluation finale (Vanhulle, 2009a). Ceci rejoint les constats faits quant aux risques de portfolios faiblement guidés qui serviraient à la fois d'outil de développement et d'instruments d'évaluation certificative (Imhof \& Picard, 2009; Tillema \& Smith, 2007). Il semble clair que, quand le principal guide d'écriture est un référentiel servant à la certification, l'étudiant se préoccupe surtout de satisfaire aux exigences plutôt que de tenter d'intérioriser les médiations et les savoirs (Francis \& Ingram Starrs, 2005).

Il nous semble important de tenir compte de ces propriétés et de proposer une médiation structurante amenant l'intériorisation de savoirs plutôt que le pilotage par des finalités. Un portfolio développemental, tel que nous le concevons, proposerait donc deux médiations: une médiation contrôlante et une médiation structurante.

Nous considérons que les portfolios offrent une médiation contrôlante se traduisant par l'intériorisation des niveaux d'autonomie de la profession. Dans l'ensemble des textes basés sur nos portfolios, nous pouvons observer une intériorisation de la médiation contrôlante. Nous relevons le passage d'une 
médiation contrôlante reposant sur les exigences du portfolio («je dois remplir cette fiche») à un contrôle par la situation («j'éprouve le besoin d'analyser cette situation») ou même par l'anticipation («je me demande ce qui arriverait si...»).

La médiation structurante faciliterait apparemment chez le sujet une intériorisation de différents savoirs. Cette intériorisation se fonderait sur le traitement de concepts et déboucherait sur une subjectivation, sur une création en propre de savoirs professionnels (Buysse \& Vanhulle, 2009). Nous constatons des progressions - variables en termes de temps et de qualité - dans l'intériorisation et la subjectivation des structures données aux savoirs au fil des écrits. Les étudiants ont tendance à pratiquer: d'abord, une stricte application des structures proposées; puis, un glissement vers une intériorisation de celles-ci au point d'un abandon total des questions proposées pour se consacrer enfin à une écriture autonome qui reprend le questionnement dans un ordre personnel. L'intériorisation progressive des médiations structurantes se dessine en même temps que la mise en discours de savoirs professionnels témoignant de l'intégration dans un réseau de significations appartenant à l'apprenant. Cette intégration peut mener à des transformations de ces significations (Vanhulle, 2005, 2009b).

Telles que nos recherches précédentes semblent l'indiquer, une démarche de subjectivation des savoirs professionnels peut résulter de l'utilisation des portfolios fondés sur l'écriture réflexive selon une vision développementale vygotskienne (Vanhulle, 2005, 2009b) et semble propice au développement professionnel (Buysse \& Vanhulle, 2009).

\section{Portfolios comme médiation contrôlante et structurante}

Afin d'atteindre cet objectif de développement impliquant la subjectivation des savoirs et des systèmes sémiotiques qui les structurent et leur donnent sens, nous nous attendons à trouver dans les dispositifs portfolio différentes caractéristiques en fonction des médiations proposées.

\section{Médiations contrôlantes}

Les apprenants devraient pouvoir se situer dans leurs apprentissages en étant amenés à faire le bilan de ce qu'ils ont appris des enseignements suivis et à évaluer leur parcours. Ces médiations amèneraient l'étudiant vers de plus grandes capacités d'autoévaluation. Toutefois, le développement d'un style d'apprentissage autorégulé ne résulte pas simplement de prédispositions du sujet, mais bien d'un ajustement du contrôle externe en fonction de celles-ci. Il existe en effet un jeu de friction et de congruence entre le contrôle de l'apprenant et celui exercé par le dispositif de formation (Vermunt \& Verloop, 1999). Le portfolio doit donc comprendre des repères permettant à un sujet qui a besoin d'un contrôle externe fort, d'intérioriser la démarche et de progressivement s'en 
distancier. Inversement, il doit aussi permettre à celui qui le désire de se donner ses propres repères. Des formulations de questions qui permettent des interprétations individualisées, des appels à l'évaluation en fonction de ses propres critères tout en proposant des repères, des dialogues ouverts avec les formateurs peuvent favoriser cette médiation.

Dans le même esprit, si l'angle d'analyse est dicté, les situations, les thèmes traités sont laissés au choix de l'étudiant. Celui-ci est également invité à autoévaluer ses actions et est laissé libre de proposer des régulations de ses actions, conceptions, croyances, tout en devant argumenter ses choix.

\section{Médiations structurantes}

Les portfolios et dispositifs de portfolios doivent selon notre point de vue proposer différentes caractéristiques favorisant les médiations structurantes.

Cela suggère notamment la prise en compte des affects pour que le sujet puisse ensuite s'en distancier en les déplaçant vers de nouvelles formes de rationalité: la mise à plat du ressenti peut ouvrir à des centrations autres que personnelles sur ce qui peut être analysé comme problématisable ou problématique dans des événements rencontrés en situation de travail. Cette prise en compte des émotions peut en effet permettre une meilleure appropriation des paramètres intervenant dans l'activité de travail sans que le sujet n'ait à craindre de jugement sur celles-ci (Boud, 2001). Il y a là une condition préalable pour entrer dans une démarche d'analyse professionnelle de la pratique. Un retour distancié sur l'expérience est ainsi rendu possible à travers une remise en contexte de celle-ci et l'identification de détails significatifs sur les circonstances. Ceci permet de comprendre ce qui s'est produit en évitant de s'en tenir à un jugement hâtif sur l'expérience.

Chaque apprenant sera par contre touché différemment par certains éléments du dispositif et il devrait pouvoir tisser des liens personnels par rapport à différents éléments de son expérience et de la formation théorique. Il est important dès lors de ne pas le contraindre à adhérer à certaines formulations imposées institutionnellement, telles celles ressortant du référentiel de compétence. Cela n'empêche pas, dans un deuxième temps, de le mettre en tension par rapport aux exigences et finalités afin de lui permettre de se situer personnellement par rapport à celles-ci. Le sujet est libre d'entrer par la partie du dispositif portfolio qui lui correspond le plus.

Un élément essentiel de la médiation structurante est le processus de questionnement que l'étudiant intériorise. Les rubriques du portfolio, mais aussi les formateurs, proposent des questionnements adaptés à différents angles d'approche. En effet, l'utilisation de "prompts», questions ou indices proposés lors de l'écriture, permettent au sujet d'activer les stratégies métacognitives nécessaires à une écriture réflexive (Hübner, Nückels \& Rentel, 2009).

Un processus de subjectivation des savoirs guidé par les dispositifs d'écriture réflexive amène à une intériorisation de la nécessité de trouver des manières de se 
les approprier. Ce mouvement est profondément lié à l'interstructuration des différents systèmes de régulation du sujet amenant à une identité professionnelle. Par exemple, une manière d'appréhender des savoirs à enseigner (objets disciplinaires) et pour enseigner (mises en œuvre didactique et pédagogique de leur enseignement et des conditions de leur apprentissage par les élèves) est d'en questionner et comprendre l'épistémie, ce qui facilite leur intériorisation.

Notre postulat est que l'écriture contribue à l'émergence de savoirs professionnels en propre dans la mesure où, à travers le procès énonciatif, le sujet façonne un discours en quête de cohérence (Vanhulle, 2009a). Les différents textes en appellent à des genres de discours spécifiques en schématisant entre l'exposé théorique ou impliqué et le récit interactif - directement lié aux situations de formation dans l'alternance cours-terrain - ou narratif autonome (Bronckart, 1996). C'est selon nous le fait d'intégrer ces différents mondes de discours pour conceptualiser, narrer, réfléchir sur l'activité et ses savoirs professionnels, qui permet à l'étudiant d'entrer dans ce que nous nommons un "genre réflexif» (Vanhulle, 2008). Les thèmes que le sujet traite, les expériences qu'il relate en vue d'en retirer des apprentissages, des pistes pour penser et agir en tant qu'enseignant, le placent, souvent, devant l'obligation d'assumer des tensions, d'ordre épistémique (confrontations à des savoirs de référence contre-intuitifs), pragmatique (confrontations à des prescriptions ou des modes d'action inattendus) et affectif (remises en cause de croyances, élucidation de valeurs, questionnements en termes d'images de soi, etc.). Le dépassement de ces tensions dans un discours qui se structure et invente sa cohérence constitue un travail de transformation en profondeur des définitions identitaires reliées aux savoirs travaillés. Ceci peut être favorisé par des outils d'écriture appartenant à des genres de discours diversifiés ou des processus de questionnement y menant. Nous pensons ici notamment à des récits d'expérience; des analyses de concepts, de notions, de systèmes théoriques; des analyses de situations, notamment à travers des incidents critiques décrits et interprétés; des réflexions documentées sur les attentes de la profession et les savoirs qui la préorganisent.

D'autre part, il peut être important d'amener l'étudiant à voir le sens de certains savoirs à enseigner et pour enseigner. Les savoirs de référence méritent aussi d'être questionnés en vue de dégager leur origine. En effet, des savoirs de nombreuses sortes influencent les prises de décisions et les explications que donne l'étudiant. Certains relèvent d'ouvrages de vulgarisation, de croyances populaires ou de prescriptions et peuvent parfois entraver la progression de l'étudiant.

Par ailleurs, il nous semble aussi primordial de travailler avec les étudiants dans le sens de la formalisation de savoirs tacites, même si ceux-ci ont leur place dans l'agir professionnel. En effet, la pratique réflexive se veut justement un moyen d'amener à expliciter les modèles d'action et donc les savoirs tacites de la pratique afin de pouvoir les transformer ou les bonifier. Un moyen est d'amener l'étudiant à analyser, à travers le dispositifportfolio, les situations d'enseignement, 
tant celles observées que celles dans lesquelles il agit. L'analyse de son expérience sur le terrain est un préalable à la constitution de savoirs d'expérience explicites.

La conception des dispositifs portfolio implique ainsi la médiation structurante nécessaire à la création des savoirs professionnels. Celle-ci consiste à problématiser les différents savoirs de la profession au niveau interpsychique afin d'être intériorisée. Pour cela, le processus même de création des savoirs professionnels doit être orchestré dans les questionnements proposés par le dispositif portfolio. Il ne s'agit pas pour autant de prétendre à une transmission des savoirs professionnels eux-mêmes ou à une quelconque taxonomie qui nierait leur caractère profondément subjectif et développemental. Il s'agit de proposer des formes de questionnement visant à soutenir un travail d'élaboration de savoirs professionnels qui repose sur l'intériorisation de leur processus même de construction (questionner, délibérer, opérer des liens, analyser les épistémies sous-jacentes, interroger les implications des concepts au regard de l'activité professionnelle, etc.). En un mot, il s'agit d'amener à d'inlassables mises en lien de la théorie avec la pratique et de l'expérience avec la théorie, afin de permettre de donner un sens qui soit fondé sur la profession et dépasse ainsi l'opinion personnelle.

Ces différents principes inspirent nos dispositifs portfolio. Ceux de Vanhulle (2005, 2009b) et collègues (Deum \& Vanhulle, 2008; Vanhulle \& Schillings, 2004) proposent une conception du portfolio fortement articulée à des interactions sociales structurées. Celles-ci s'accompagnent systématiquement de propositions de mise en texte de savoirs professionnels. Le portfolio se subdivise dans des rubriques incitant à mobiliser quatre axes de réflexivité, centrés sur: le sujet biographique et son parcours propre d'élève puis d'enseignant; l'autoévaluation de son parcours, des compétences et savoirs acquis; la manipulation critique et créative de concepts et modèles théoriques issus de la recherche en sciences de l'éducation; l'autoguidage des objectifs et des actions en milieu de pratiques. Ces quatre axes sont reliés dans des textes de synthèse, puis dans un récit final de formation qui clôture le processus, le portfolio étant organisé par un sommaire commenté du choix des traces d'apprentissage recueillies au fil du temps et des textes réflexifs produits. Ce récit et le sommaire final du portfolio sont les seuls travaux certifiés en fin de formation. Tout au long du parcours, des rétroactions des formateurs mais aussi des présentations régulières du portfolio devant les pairs (tous les textes ou traces n'étant pas obligatoirement dévoilés, ni aux formateurs ni aux condisciples), sont destinées à proposer des régulations externes et potentiellement à soutenir l'autorégulation et ce que nous avons nommé plus haut la réflexivité structurante. Selon ce même but, le dispositif est constamment ajusté en fonction des questionnements, difficultés ou contenus de savoirs que le groupe et chaque individu mettent en avant - ceci de manière à travailler au mieux en fonction des "zones de développement proche» des sujets, et à transformer les séquences de formation en lieux d'émergence d' "expériences cruciales» (Vygotsky, 1997) qui peuvent se poursuivre dans l'écriture, favorisant le remaniement des modes de penser, de faire, d'interpréter, de se penser comme profes- 
sionnel de l'apprentissage scolaire. La finalité d'une telle ingénierie est clairement que l'intériorisation des contraintes, offres de significations et de procédures apportés par le dispositif puissent être saisies par les sujets comme des opportunités au service de leur développement.

Dans la partie qui suit, nous allons examiner de manière plus précise un dispositif portfolio conçu par Buysse pour illustrer en quoi celui-ci organise volontairement des médiations, entre médiations contrôlantes et structurantes.

\section{Portfolio de développement comme instrument au centre de l'élaboration des savoirs profes- sionnels}

Buysse (en préparation) propose un portfolio ${ }^{5}$ conçu selon deux grands outils réflexifs: une partie «suivi des études», à visée de médiation contrôlante, et une partie "portfolio de développement», à visée principalement structurante. Les traces sont ici vues comme des illustrations de la démarche et l'écriture réflexive est guidée à travers des questionnements.

La construction du portfolio amène l'étudiant à faire des liens entre différentes rubriques et comprendre la structure de la formation ainsi que le mécanisme d'alternance. Si le questionnement est fortement guidé, le choix des sujets est laissé libre aux étudiants, ainsi que la priorité accordée à telle ou telle partie du portfolio. En effet, des redondances permettent à l'étudiant d'aborder le portfolio par différentes entrées. Notons que différentes présentations et variantes du même questionnement sont proposées pour chaque rubrique. Nous pourrions dire que le portfolio est, dans ce cas, une collection d'instruments mis à disposition de l'étudiant avec comme finalité: d'une part, la prise de contrôle de ses apprentissages et de son développement professionnel et, d'autre part, l'intériorisation d'un processus de création de savoirs professionnels.

La partie «Suivi des études» comprend des rubriques portant, entre autres, sur l'évolution des représentations quant à la profession et son insertion dans celleci, les objectifs généraux de formation que l'étudiant se fixe, les savoirs qu'il veut pouvoir acquérir à travers les différents cours et ceux qu'il pense s'être effectivement appropriés, les synthèses de ses entretiens de formation en stage et le suivi de l'atteinte des objectifs de formation, l'évolution de son sujet de mémoire final. Dans chacune de ces rubriques, l'étudiant est invité à faire des liens avec d'autres parties du portfolio ou entre la théorie et la pratique.

La partie «Développement professionnel» comprend des processus guidés de réflexion sur des événements. Ce questionnement est basé sur les étapes de Schön (1983) et propose pour chaque situation, ou action en situation, d'identifier les caractéristiques qui singularisent celle-ci; de formuler une problématisation; de mettre en lien des savoirs de référence de différentes sources permettant de comprendre les raisons du succès ou de l'échec des actions entreprises; de 
proposer de nouvelles pistes pour l'agir. Les questionnements proposés affinent toutefois ces étapes en introduisant systématiquement des interrogations sur les savoirs de référence, les autres exemples issus de l'expérience subjective, les savoirs à enseigner impliqués, afin d'amener à la prise en compte de la complexité de la profession.

Là aussi, plusieurs rubriques sont proposées: analyse de situation ou d'idées ne mettant pas en jeu l'étudiant, analyse de savoirs, analyse des actions en situation de l'étudiant. Des mises en lien avec d'autres parties du portfolio ou entre théorie et pratique sont constamment sollicitées.

Une analyse du parcours de formation est demandée et amène progressivement vers une synthèse dans laquelle l'étudiant est invité à tenter de formuler des savoirs professionnels en les référant, sur l'ensemble de sa formation, à des expériences et à des apports théoriques ou autres. Afin de permettre à l'étudiant d'approfondir sa réflexivité, il est amené à s'interroger sur ce qu'il prend en compte lors de ces différentes analyses.

Vers la fin du travail avec le portfolio, dans une volonté de permettre la "présentation critique du portfolio» qui est un des volets de l'examen de fin d'études, un lien est demandé entre les compétences exigées selon le référentiel de formation et les savoirs professionnels de l'étudiant. Il élabore ainsi un texte réflexif et une présentation tissant des liens entre les événements importants de sa formation, les savoirs professionnels développés et les compétences.

Le dispositif implique l'interaction autour du portfolio d'un ensemble d'intervenants de pratique réflexive tels le mentor ${ }^{6}$, le superviseur, les formateurs terrains (Truffer Moreau, 2009). Il nous semble en effet indispensable que le portfolio soit totalement intégré dans le dispositif de formation afin que les apprenants ne perçoivent pas les portfolios comme étant une obligation sans intérêt (Imhof \& Picard, 2009). Les mentors accompagnent les étudiants dans leur processus de portfolio en leur permettant de mettre le doigt sur des forces et des faiblesses, donc de leur donner une pleine force de médiation contrôlante (Riggs, Sandlin, Scott, Childress \& Mitchell, 1997). En outre, ils interviennent également comme formateurs et font des liens entre la théorie et la pratique (Brockbank \& McGill, 2006). Ils peuvent ainsi guider l'étudiant vers des approches qu'il n'aurait pas sélectionnées de lui-même (Orland-Barak, 2005).

L'étudiant est invité à partager ses analyses avec ses collègues mais aussi avec les formateurs en dehors de tout processus d'évaluation. Un cours assure le suivi $\mathrm{du}$ portfolio durant les trois ans de formation en expliquant les bases théoriques et en explicitant les démarches incluses dans chacun des outils. Le travail sur le portfolio est évalué au niveau de l'implication de l'étudiant une fois par année et crédité afin de valoriser le travail important exigé.

L'utilisation d'un journal de bord de formation (Truffer Moreau \& Périsset Bagnoud, 2007) est incluse dans le dispositif en tant que prise de notes et premier jet de réflexivité 7 . Dans son utilisation conjointe avec le portfolio il reste totalement privé et fait l'objet d'une faible structuration. 
La pratique réflexive est également au centre des entretiens de formation tant avec les superviseurs qu'avec les formateurs terrain, en insistant autant sur la réflexivité que sur les apports formatifs, théoriques ou pratiques des intervenants. Dès le début de la formation, il ne s'agit pas pour l'étudiant de faire preuve de réflexivité au sens large mais de s'approprier une des manières proposées de penser sa pratique.

\section{Discussion}

Dans ce dispositif portfolio, deux médiations sont clairement à l'œuvre: une médiation contrôlante visant à permettre à l'étudiant d'intérioriser le contrôle de son apprentissage mais aussi de s'approprier les éléments à contrôler dans sa profession; et une médiation structurante qui amène une création des savoirs professionnels et certains raisonnements propres aux enseignants afin de pouvoir intérioriser les savoirs à enseigner et les savoirs pour enseigner.

Nous pensons que la plupart des dispositifs portfolio se situent en tant que médiation contrôlante, favorisant le développement de l'apprentissage autorégulé (Tanner, Longayroux, Beijaard \& Verloop, 2000) en passant par l'autoévaluation (Mottier Lopez \& Vanhulle, 2009). Il s'agit là à notre avis du résultat d'une lente intériorisation de la médiation contrôlante du portfolio tel que les différents formateurs le conçoivent et l'accompagnent. Néanmoins, même dans des portfolios mettant l'accent sur l'autoévaluation et donc sur la médiation contrôlante, un effet "secondaire» semble être la facilitation de la construction de savoirs par les étudiants (McFarland, 1998). Des portfolios proposant de manière plus accentuée des médiations structurantes devraient d'autant plus faciliter la construction des savoirs professionnels.

D'après nos recherches, la qualité de cette élaboration ne dépend pas seulement de la fonction en soi structurante de l'écriture. On observe à cet égard des progressions chez la majorité des étudiants, y compris ceux qui sont au départ peu compétents sur le plan scriptural: la qualité de l'élaboration dépend de leur engagement dans le double processus qui combine les interactions sociales inhérentes au dispositif et la construction d'un portfolio. D'autant plus que celui-ci est utilisé non pas comme un simple instrument de présentation de textes adaptés à des attentes académiques, mais comme un outil sémiotique qui aide à penser, revenir sur ses actions, se positionner et se transformer depuis une posture "étudiant» vers une posture "professionnelle» en quête d'expertise et d'argumentation sur ce qui fonde l'activité enseignante (Vanhulle, 2009c, 2009d).

Nous en concluons que des dispositifs mettant en avant des médiations structurantes inhérentes à la profession permettent de favoriser le développement professionnel des apprenants (Buysse \& Vanhulle, 2009, august), quoique des recherches plus fines seraient nécessaires afin de s'assurer que les médiations 
structurantes proposées rejoignent vraiment une épistémie de la profession enseignante.

Notons également que l'introduction d'un portfolio ou d'un nouveau portfolio n'est que le début d'un processus de changement pour l'institution (Craig, 2003), particulièrement s'il s'agit d'un portfolio offrant une médiation structurante forte car celle-ci implique un raisonnement lié à la formation et à la profession dans son ensemble. Pour les formateurs, cela implique de travailler avec les étudiants sur les tensions que l'intériorisation des médiations provoquent en eux, de manière à ce qu'ils s'engagent dans le processus et de pratiquer un accompagnement en termes de didactique des savoirs professionnels. Ceci suppose de relier les voies a priori séparées du développement: celle de l'appropriation fine de savoirs de référence externes établis pour la profession et celle de l'expérience en milieu de travail.

\section{Notes}

1 Haute École pédagogique du Valais et Université de Genvève, Section des sciences de l'éducation, groupe de Recherche "Théorie, Action, Langage et Savoirs (TALES)» dirigé par Prof. S. Vanhulle

2 La réflexivité contrôlante correspond à la prise de conscience du processus de régulation.

3 Développement d'un style d'apprentissage autorégulé ou Self regulated learning style (voir e.a. Boekaerts \& Niemivirta, 2005)

4 La réflexivité structurante correspond à la prise en compte de différents savoirs et informations afin de: réorienter le processus de régulation; consolider la régulation aboutie et permettre ainsi l'appropriation de nouveaux savoirs; permettre la restructuration des savoirs préexistants chez le sujet.

5 Ce portfolio a été introduit à la Haute école pédagogique du Valais en 2008, suite à une évaluation des dispositifs (Périsset Bagnoud, Andrey-Berclaz, Steiner \& Ruppen, 2006) et une recherche sur les dispositifs existant dans l'institution (Périsset Bagnoud \& Buysse, 2008).

6 Le mentorat à la HEP-VS fonctionne de la manière suivante: chaque étudiant se voit attribué pour la durée de ses études un mentor qui est enseignant à la HEP. Il a comme fonction principale de l'amener à faire des liens entre théorie et pratique, ainsi que de l'aider à entrer et évoluer dans la démarche portfolio.

7 Notons que les formateurs de terrain, appelés praticiens formateurs, sont formés à l'utilisation du journal de bord et du portfolio. Ils bénéficient en effet des mêmes dispositifs dans leur propre formation de trois ans. Ceci leur permet, entre autres, de mieux accompagner les étudiants (Truffer Moreau, 2009).

\section{Références bibliographiques}

Antonek, J. L. (1997). The student teacher portfolio as autobiography: Developing a professional identity. Modern Language Journal, 81, (1), 15-27.

Berrill, D. P. \& Whalen, C. (2007). "Where are the children?»: Personal integrity and reflective teaching portfolios. Teaching and Teacher Education, 23, (6), 868-884.

Boekaerts, M. \& Niemivirta, M. (2005). Self-regulated learning: Finding a balance between learning goals and ego-protective goals. In M. Boekaerts, P. R. Pintrich \& M. Zeidner (Éd.), Handbook of self-regulation (pp. 417-450). Burlington, MA: Elesevier.

Boud, D. (2001). Using journal writing to enhance reflective practice. New Directions for Adult and Continuing Education, 2001, (90), 9-17. 
Brockbank, A. \& McGill, I. (2006). Facilitating reflective learning through mentoring and coaching. London: Kogan Page.

Bronckart, J.-P. (1996). Activité langagière, textes et discours. Pour un interactionnisme sociodiscursif. Neuchâtel: Delachaux et Niestlé.

Buysse, A. (2008, octobre). Entre apprentissage et développement: les médiations internalisantes du signe, de l'outil et de la forme. Communication présentée au séminaire de recherche Langage, action, formation. Prof. Bronckart, Université de Genève.

Buysse, A. (2009). Médiations contrôlantes et structurantes: une base pour penser la formation. Revue suisse des sciences de l'éducation, 3, 585-602.

Buysse, A. (sous presse a). Les mécanismes inductifs et déductifs dans la genèse des savoirs des enseignants en formation. In Y. Lenoir \& P. Maubant (Éd.), Quand la formation des adultes sinvite au débat sur la formation professionnelle des enseignants. Ottawa: Presses Universitaires.

Buysse, A. (sous presse b). Une modélisation des régulations et de la médiation dans la construction des savoirs professionnels des enseignants. In P. Maubant \& S. Martineau (Éd.), Ancrages organisateurs de la pratique enseignante et logiques d'action: fondements des pratiques professionnelles des enseignants. Ottawa: Presses Universitaires.

Buysse, A. (en préparation). Formation des enseignants: un dispositif pour le développement professionnel.

Buysse, A. \& Vanhulle, S. (2009). Écriture réflexive et développement professionnel: quels indicateurs? Questions Vives 5, (11), 225-245.

Buysse, A. \& Vanhulle, S. (2009, august). Portfolio as mediation on levels of reflexivity and regulation: A way to enhance professional development in teacher education. Paper presented at the symposium on portfolio in teacher education. M. Imhof \& M. Gläser-Zikuda (Prés.). EARLI 2009, Amsterdam.

Costantino, P., De Lorenzo, M. \& Kobrinski, E. (2002). Developing a professional teaching portfolio. Boston: Pearson.

Craig, C. J. (2003). School portfolio development: A teacher knowledge approach. Journal of Teacher Education, 54, (2), 122-134.

Deum, M. \& Vanhulle, S. (2008). Le portfolio, un outil de professionnalisation des enseignants (Cahier de la section des sciences de l'éducation, Vol. 116). Université de Genève.

Doolittle, P. (1994). Teacher portfolio assessment. ERIC/AE Digest (No. EDO-TM-94-07). Washington, DC.

Fenstermacher, G. D. (1994). The knower and the known: The nature of knowledge in research on teaching. Review of Research in Education, 20, 3-56.

Fenstermacher, G. D. \& Sanger, M. (1998). What is the significance of John Dewey's approach to the problem of knowldege? The Elementary School Journal, 98, (5), 467-478.

Foster, B. R., Walker, M. \& Song, K. H. (2007). A beginning teaching portfolio handbook. Upper Saddle River, NJ: Pearson.

Francis, D. \& Ingram Starrs, L. (2005). The labour of learning to reflect. Teachers and Teaching: Theory and Practice, 11, (6), 541-553.

Hofstetter, R. \& Schneuwly, B. (Éd.). (2009). Transformation des savoirs de référence des professions de l'enseignement et de la formation (Collection Raisons éducatives). Bruxelles: De Boeck.

Hübner, S., Nückels, M. \& Renkl, A. (2009). Writing learning journals: Instructional support to overcome learning-strategy deficits. Learning and Instruction, 20, (1), 1-12.

Imhof, M. \& Picard, C. (2009). Views on using portfolio in teacher education. Teaching and Teacher Education, 25, 149-154.

Karpov, Y. V. (2003). Internalization of children's problem solving and individual differences in learning. Cognitive Development, 18, 377-398.

Karpov, Y. V. \& Haywood, H. C. (1998). Two ways to elaborate Vygotsky's concept of mediation. American Psychologist, 53, (1), 27-36. 
McFarland, K. P. (1998). Promoting reflective practices in pre-service teacher education: An activity on stages of concern. Shippensburg, PA: Shippensburg University.

Mottier Lopez, L. \& Vanhulle, S. (2009). Portfolios et entretiens de coévaluation: des leviers de la professionnalisation des jeunes enseignants. In G. Baillat, C. Thélot, L. Paquay \& J.M. de Ketele (Ed.), Evaluer pour former, quelles démarches, quels outils (pp. 143-159) Bruxelles: De Boeck.

Orland-Barak, L. (2005). Portfolios as evidence of reflective practice: What remains 'untold'. Educational Research, 47, (1), 25-44.

Paris, S. G. \& Ayres, L. R. (1994). Becoming reflective students and teachers with portfolios and authentic assessment. Washington D.C.: APA American Psychological Association.

Périsset Bagnoud, D. \& Buysse, A. (2008). La pratique réflexive entre intentions et situations de formation. In L. Mottier Lopez, Y.-E. Dizerens, G. Marcoux \& A. Perréard Vité (Éd.), Entre la régulation des apprentissages et le pilotage des systèmes: évaluations en tension. Actes du 20e colloque ADMEE- Europe, Université de Genève. Consulté le 20 janvier 2009 dans https://plone.unige.ch/sites/admee08/symposiums/j-s5/j-s5-4/

Périsset Bagnoud, D., Andrey-Berclaz, M., Steiner, E. \& Ruppen, P. (2006). Evaluation de la formation initiale de la HEP-VS. Cinq Rapports: les étudiant-es; les enseignant-es; les praticiens-formateurs; les entretiens; synthèse et comparaison. St-Maurice et Brig: Haute École Pédagogique du Valais.

Schön, D. A. (1983). The reflective practitioner: How professionals think in action. Aldershot: Ashgate.

Schön, D. A. (1987). Educating the reflective practitioner: Toward a new design for teaching and learning in the professions. San Francisco: Jossey-Bass.

Tanner, R., Longayroux, D., Beijaard, D. \& Verloop, N. (2000). Piloting portfolios: Using portfolios in pre-service teacher education. ELT journal, 54, (1), 20-30.

Tillema, H. \& Smith, K. (2007). Portfolio appraisal: In search of criteria. Teaching and Teacher Education, 23, 442-456.

Truffer Moreau, I. (2009). La formation des PF à l'encadrement des stagiaires. St-Maurice et Brig: Haute École Pédagogique du Valais.

Truffer Moreau, I. \& Périsset Bagnoud, D. (2007). Écrire pour se former. Un outil de formation et d'autoévaluation: le journal de bord des formateurs de terrain. Mesure et évaluation en éducation, 30, (1), 79-96.

Van der Veer, R. (1998). From concept attainment to knowledge formation. Mind, Culture, and Activity, 5, (2), 89-94.

Vanhulle, S. (2005). How future teachers develop professional knowledge through reflective writing in a dialogical frame. Educational studies in language and littérature, 5, 287-314.

Vanhulle, S. (2008). Au coeur de la didactique professionnelle, la subjectivation des savoirs. In P. Pastré \& Y. Lenoir (Éd.), Didactique professionnelle, didactique des savoirs professionnels (pp. 227-254). Toulouse: Octarès.

Vanhulle, S. (2009a). Accompagner la construction des savoirs professionnels dans l'écriture réflexive. Travail et apprentissages, Revue de Didactique Professionnelle, 3, 63-75.

Vanhulle, S. (2009b). Des savoirs en jeu au savoir en je. Cheminements réflexifs et subjectivation des savoirs chez de jeunes enseignants en formation. Berne: Peter Lang.

Vanhulle, S. (2009c). Dire les savoirs professionnels: savoirs de référence et logiques d'action. In R. Hofstetter \& B. Schneuwly (Éd.), Savoirs en (trans)formation. Au cour de l'enseignement et de la formation (Collection Raisons éducatives) (pp. 245-264). Bruxelles: De Boeck.

Vanhulle, S. (2009d). Évaluer la professionnalité émergente: un pari entre cadres contraignants et tensions formatives. In L. Mottier Lopez \& M. Crahay (Éd.), Évaluations en tension: entre la régulation des apprentissages et le pilotage des systèmes (pp. 165-180). Bruxelles: De Boeck. 
Vanhulle, S. (sous presse). Savoirs professionnels et construction sociodiscursive de l'agir. Bulletin Suisse de Linguistique Appliquée.

Vanhulle, S. \& Schillings, A. (2004). Former des enseignants compétents en lecture-écriture: interactions sociales, portfolio, et écriture réflexive. Bruxelles: Labor.

Vermunt, J. D. \& Verloop, N. (1999). Congruence and friction between learning and teaching. Learning and Instruction, 9, (3), 257-280.

Vygotsky, L. (1978). Mind in society: The development of higher psychological processes. London: Harvard University Press.

Vygotsky, L. (1997). On psychological systems (R. Van der Veer, trad.). In R. W. Rieber \& J. Wollock (Éd.), The collected works of L.S. Vygotsky (Vol. 3): Problems of the theory and history of psychology (pp. 91-108). New York: Plenum.

Wood, E. \& Bennett, N. (2000). Changing theories, changing practice: Exploring early childhood teachers' professional learning. Teaching and Teacher Education, 16, (5-6), 635647.

Mots clés: Portfolio, médiation, médiation structurante, médiation contrôlante, développement

\section{Das Portfolio als eine kontrollierende \& strukturierende Vermittlung von professionellem Wissen}

\section{Zusammenfassung}

In der Perspektive von Vygotskij, die auch wir einnehmen, ist das Portfolio ein Werkzeug, das nicht nur eine Vermittlung der Kontrolle von Lernprozessen, sondern auch eine Vermittlung von Wissensprozessen erlaubt. Wir erachten in diesem Sinne das Portfolio als eine kontrollierende und strukturierende Vermittlung. Wir überprüfen, wie durch das Portfolio ermöglicht wird, zunächst die Aufbauprozesse von - auf Referenz- und subjektivem Erfahrungswissen basierendem - professionellem Wissen sichtbar zu machen, und anschliessend dieses zu verinnerlichen. Unsere Äusserungen werden illustriert anhand der Beschreibung unseres Interventions-Portfolios im Kontext unserer Institution für die Lehrpersonenausbildung.

Schlagworte: Portfolio, Vermittlung, Professionelles Wissen, Lehrpersonenausbildung, Entwicklung 


\section{II portfolio: una mediazione controllata e strutturata dei saperi professionali}

\section{Riassunto}

Secondo la prospettiva vygotskiana, che abbiamo fatto nostra, il portfolio è uno strumento che permette non solo una mediazione controllata degli apprendimenti, ma anche una mediazione delle procedure conoscitive. Ne risulta una mediazione attiva, controllata a strutturante. Nell'articolo analizziamo come il portfolio permette di rendere visibili dapprima e di interiorizzare in seguito i processi di creazione dei saperi professionali derivanti dalle discipline di riferimento e dall'esperienza. L'illustrazione avviene sulla base dei nostri interventi all'istituto per la formazione degli insegnanti.

Parole chiave: Portfolio, mediazione, formazione degli insegnanti, saperi professionali, sviluppo

\section{Portfolio as controlling and structuring mediation of professional knowledge}

\section{Summary}

According to our Vygotskian perspective, a portfolio is a tool that mediates not only the extent to which the students control their own learning, but also a mediation of the procedures linked to knowledge. We consider portfolio as both a controlling and a structuring mediation. We examine how it could enable first to see, and then to internalise, the creation process of professional knowledge based on referential and experiential knowledge at the disposal of the student. Following a short definition of the portfolio, the mediations are described as well as the peculiar points about professional knowledge. To illustrate our findings we describe two different sets of intervention-portfolio developed in the context of our teacher training institutions.

Key words: Portfolio, mediation, professional knowledge, teacher training, development 